\title{
Path analysis of work
}

conditions and work-family

spillover as modifiable

workplace factors associated

with depressive

symptomatology

Renée-Louise Franche, ${ }^{*,+, 1}$ Alysha Williams, ${ }^{2}$ Selahadin Ibrahim, ${ }^{1}$ Sherry L. Grace, ${ }^{3}$

Cam Mustard, ${ }^{1}$ Bruce Minore ${ }^{4}$ and Donna E. Stewart ${ }^{5}$

\section{Summary}

Background: Literature pertaining to the relationship between workplace factors and depression has been compartmentalized: work conditions, family conditions, and work-family balance have been studied separately as predictors of depressive symptoms but not concurrently.

Objective: Work conditions and work-family spillover were considered concurrently as modifiable workplace factors associated with depressive symptomatology, while controlling for confounding socio-economic factors.

Methods: This cross-sectional study involved 218 female health care workers who completed a survey assessing work conditions [Effort-Reward Imbalance (ERI) scale and Job Content Questionnaire (JCQ)], work-family balance (work-family spillover scale), sociodemographic information, and depressive symptoms [Center for Epidemiological Studies Depression (CES-D) scale]. Results: Path analysis supported the presence of a direct relationship between depressive symptoms and high effort-reward imbalance, high negative work-family spillover, low positive familyto-work spillover, and low education. The indirect effect of low support from work was mediated by negative work-to-family spillover and high effort-reward imbalance. The indirect effect of high effort-reward imbalance was mediated by increased negative work-to-family spillover. The indirect effect of having children 18 years or younger was mediated by decreased positive family-towork spillover. An indirect effect of low education was mediated by high effort-reward imbalance and high negative work-to-family spillover.

\footnotetext{
* Correspondence to: Renée-Louise Franche, Institute for Work \& Health, 481 University Avenue, Suite 800,
} Toronto, ON, M5G 2E9, Canada. Tel: 416-927-2027 (2222).

†E-mail: rfranche@iwh.on.ca 
Conclusions: The association between work conditions and depressive symptomatology is mediated by increased negative work-to-family spillover. The impact of having young children is mediated by decreased positive family-to-work spillover.

\section{Key Words}

work conditions; depression; work-family balance

\section{Introduction}

Depression in the workplace has become an emerging focus in the occupational health field as a dominant cause of disability. It is estimated that 5.6 per cent of Canadian workers (Wang \& Patten, 2001) experience at any point in time clinically significant levels of depression. Depression is associated with activity limitations, including occupational activities (Broadhead, Blazer, George, \& Tse, 1990; Stephens \& Joubert, 2001). It is also associated with a high level of health service utilization (Johnson, Weissman, \& Klerman, 1992; Stephens \& Joubert, 2001) and high levels of work absenteeism (Goldberg \& Steury, 2001; Simon et al., 2001). Human resources and employee assistance personnel are now aware of the importance of addressing workplace risk factors for depression, and the need to expand research in this area has been highlighted (Baba, Jamal, \& Tourigny, 2003).

The literature pertaining to workplace factors and depression appears compartmentalized in that studies either examine work conditions, family conditions, or work-family balance, but rarely are these domains examined concurrently. There is a need for a more integrated approach to the understanding of how workplace factors impact on depressive symptoms.

The objective of the current study was to develop and test a model of associations between work conditions, work-family balance, and depressive symptoms in a sample of health care workers, using path analysis. This group of workers generally has a high prevalence of psychological distress (Bellerose, Lavallée, Chénard, \& Levasseur, 1995; Préville, Beauchemin, \& Potvin, 1991; Sauter, Murphy, \& Hurrell, 1990; Wall et al., 1997).

First the relationship between work-family balance and depression will be discussed. This will be followed by a discussion of how the constructs of the two main models of occupational stress-the Demand-Control (DC) model (Karasek, 1979; Karasek et al., 1998) and the Effort-Reward Imbalance (ERI) model (Siegrist \& Peter, 1996)-impact depressive symptoms.

\section{Work-family balance and depressive symptomatology}

The relevance of work-family balance to mental health, and to depression in particular, is gaining more attention. Certain basic concepts in the area of work-family balance need to be defined (Frone, 2003). Work-family spillover refers to the concept that activities in one domain have an impact on the other. The study of work-family spillover is enhanced by examining both directions of work-family spillover, that is work-tofamily spillover, and family-to-work spillover, and by the recognition that spillover can be positive (facilitation) or negative (conflict) (Grzywacz \& Bass, 2003; Grzywacz \& Marks, 2000; Grzywacz \& Dooley, 2003; Kelloway, Gottlieb, \& Barham, 1999; Voydanoff, 2004). In keeping with this approach, the relationship between work-family balance and depressive symptomatology will now be discussed.

One cross-sectional study (Frone, Russell, \& Barnes, 1996) found both work-to-family and family-to-work conflict to be significantly associated with depressive symptoms (Radloff, 1977). However, the facilitative effects of family-to-work and work-to-family spillover were not assessed in the study. A second cross-sectional study (Grzywacz \& Dooley, 2003) found family-towork conflict, family-to-work facilitation, and to a lesser degree work-to-family conflict, all significantly associated with depression (Kessler, Andrews, Mroczek, Ustun, \& Wittchen, 1998). Both studies controlled for the confounding effects of age, gender, race, education, marital status, and income. With the exception of working hours (Frone et al., 1996), the impact of 


\section{Work conditions, work-family spillover, and depressive symptomatology}

working conditions was neither examined nor controlled for in these two studies.

Few studies address more than one of the domains of work, family, and work-family characteristics, and their relationship with depressive symptoms. Among the studies which did address more than one domain of interest, a crosssectional study (Major, Klein, \& Ehrhart, 2002) showed how work and family demands impacted working hours, which were positively associated with work interfering with family, which in turn was associated with higher levels of depressive symptoms and somatization. In this study, there was no assessment of family-to-work conflict, family-to-work facilitation, or work-to-family facilitation.

The cross-sectional nature of these earlier studies precludes any inference of causality. To the authors' knowledge, only one study (Frone, Russell, \& Cooper, 1997) used a longitudinal design and showed that after controlling for baseline levels of outcomes, family-to-work conflict was predictive of higher levels of depressive symptoms. These results highlight the importance of addressing the role of work-family balance in the development of depressive symptoms.

\section{The Demand-Control (DC) model and the Effort-Reward Imbalance (ERI) model}

The two main models of work conditions will now be described, and their impact on depressive symptomatology briefly reviewed. It is important to note that these two models do not incorporate work-family balance aspects. The DC model (Karasek, 1979; Karasek et al., 1998) addresses the two dimensions of demand and control in the work environment. These two dimensions can be viewed as creating four quadrants (high/low demand with high/low control). The ERI model (Siegrist \& Peter, 1996) is based on the proposition that psychological stress occurs when there is an imbalance between what is invested in one's work and what is gained. While some overlap exists between the DC and ERI models, namely between the DC demands and ERI effort dimensions, the models differ in their general focusthe DC model is focused on the job task demands while the ERI model addresses a broader array of work characteristics. Most notably, the ERI incorporates factors associated with self-esteem such as status control, job security, and rewards, and macro-economic factors such as job security.
Several approaches have been used to interpret results obtained when using the DC model: (1) the strain model posits that individuals with high demands and low control experience the highest levels of stress, as compared to the three other quadrants; (2) the buffering model of the strain model explicitly posits an interactive process where control buffers the effects of high demands; (3) the iso-strain model incorporates the dimension of social support received at work, in that individuals in high demands/low control/low social support are believed to be the ones experiencing the highest levels of work stress; (4) the buffering model of the iso-strain model proposes that more control and more social support buffer the impact of high demands.

With regards to the relationship between the DC model and psychological distress, an extensive review suggests a strong relationship between the DC model and psychological distress when the strain and iso-strain models are used (van der Doef \& Maes, 1999). In more recent studies, the demand and control subscales have not been combined into strain and iso-strain and instead the contributions of each subscale to depressive symptomatology have been examined separately. In a large French cohort study, high psychological demands, low decision latitude, and low social support at work significantly predicted depressive symptoms in both men and women, even when controlling for confounding variables (Niedhammer, Goldberg, Leclerc, Bugel, \& David, 1998; Paterniti, Niedhammer, Lang, \& Consoli, 2002). As well, in the longitudinal Whitehall study, the decision authority subscale was significantly associated with depressive symptoms (Stansfeld, Fuhrer, Shipley, \& Marmot, 1999). In addition, both psychological and physical demands subscales, when analyzed separately, have been significantly associated with depression in a cross-sectional study (MausnerDorsch \& Eaton, 2000).

It is important to consider the impact of social support from work on employee health. Social support refers to support from supervisors and coworkers, and to the presence of positive interactions at work. Previous longitudinal studies have found a robust relationship between low social support from work and depressive symptomatology (Dormann \& Zapf, 1999; Stansfeld et al., 1999), and low social support and general psychological distress (Bourbonnais, Comeau, \& Vézina, 1999).

Studies examining the relationship between the ERI and psychological well-being have compared 
or juxtaposed the contributions of the ERI and the DC models. These studies did not examine depressive symptomatology as an outcome but focused on measures of general psychological well-being. The studies support independent cumulative effects of both models. In one large cross-sectional study (de Jonge, Bosma, Peter, \& Siegrist, 2000), when both models were entered simultaneously, high efforts and low rewards were stronger predictors of poor well-being than low job control. In the longitudinal Whitehall study, high demands and high ERI were risk factors for future psychiatric disorder, while social support and control were protective factors (Stansfeld et al., 1999).

Taken together, results of these earlier longitudinal studies suggest that much is gained by examining the contributions of both the DC and ERI models concurrently.

\section{Summary}

There is a need for studies examining the concurrent impact of work conditions and workfamily balance on mental health of workers. Compartmentalizing the impact of work-family balance and work conditions leads to partial answers and can not assess the relative impact of either group of factors, or the relationship between them. By examining both work conditions and work-family balance as predictors of mental health outcomes, a more comprehensive model of how work affects mental health can begin to emerge.

\section{Hypotheses}

Path analysis was used to develop and test a model of the relationships between work conditions, work-family balance variables, and the outcome of depressive symptomatology. This hypothesized model was based on the following hypotheses:

(1) Based on the reviewed literature, it is hypothesized that direct relationships between the ERI ratio and depressive symptomatology, between JCQ strain (control/demand) and depressive symptomatology, and between social support received at work (JCQ) and depressive symptomatology.
(2) It is hypothesized that indirect effects of the ERI, the JCQ strain, and the JCQ social support with depressive symptomatology, would be mediated by the four spillover constructs: negative work-tofamily spillover (NWFS), positive work-tofamily spillover (PWFS), negative familyto-work spillover (NFWS), and positive family-to-work spillover (PFWS). Negative spillover would be associated with higher levels of depressive symptomatology, while positive spillover would be associated with lower levels of depressive symptomatology.

(3) It is hypothesized that lower education and lower income would be directly associated with depressive symptomatology (Lorant et al., 2003). Also examined is the relationship between other socio-economic/health variables (age, marital status, presence of children, presence of children of 18 years or younger, number of hours worked, pregnancy status) and depressive symptomatology.

(4) It is hypothesized that having children and/or having children 18 years of age or younger would be directly related to higher levels of NWFS and NFWS, and lower levels of PWFS and PFWS, which in turn would mediate the effects on depressive symptomatology.

\section{Method}

\section{Participants}

A sample of 243 female employees working in a range of occupations in three Ontario health care centers participated in the study ${ }^{1}$. Of the 243 participants, 218 (90 per cent) had complete data for all variables considered in the study. All analyses were conducted using this sample of 218 participants.

\footnotetext{
${ }^{1}$ Both women and men were recruited. However, only 18 male employees participated in the study. The male employees' level of depression were significantly higher than in the study sample and than what would be expected in the general male population, suggesting a strong selection bias. Given the skewed distribution of the depression scores, the fact that they could not be considered as representative of the population of interest, and given the small cell size, they were excluded from subsequent analyses.
} 


\section{Work conditions, work-family spillover, and depressive symptomatology}

Participants were recruited from three acute health care centers located in three different cities. One hundred and twenty-nine workers were from one health care center of 14,500 employees in a large metropolitan area; 47 workers were from a health care center of 11,000 employees in a medium size city; 42 workers were from a smaller center of 1900 employees in a small northern city located in a largely rural area. One aspect of the study involved a focus on pregnant employees and consequently they are over-represented in the current sample (17.4 per cent are pregnant).

Sample characteristics were the following. Participants' mean age was 39.43 (standard deviation, $\mathrm{SD}=7.91$ ), 69.3 per cent were parents, 22.0 per cent had one child, 35.8 per cent had two children, 11.5 per cent had three children or more, 76.6 per cent were partnered (married or common-law), 11.5 per cent had completed high school or had some university or college education (but not completed), 66.5 per cent had completed a college or university degree, 22.0 per cent had graduate education or a professional degree. Personal income was distributed in the following way: 7.4 per cent earned less than $\$ 29,999,20.3$ per cent between $\$ 30,000$ and 39,999, 15.2 per cent, between $\$ 40,000$ and 49,999, 24.9 per cent between $\$ 50,000$ and $\$ 59,999,32.3$ per cent above $\$ 60,000$. Family income was distributed in the following way: 19.5 per cent under $\$ 59,999$, 21.8 per cent between $\$ 60,000$ and $79,999,16.2$ per cent between $\$ 80,000$ and $99,999,19.9$ per cent between $\$ 100,000$ and $\$ 119,999,22.7$ per cent above $\$ 120,000$. Average number of hours worked per week was 37.63 hours $(S D=8.48)$.

Participants were asked to provide their occupation in their own words. This was subsequently coded in two ways. First, the self-reported descriptions were categorized in one of the following categories of area of work: administration (28.0 per cent), nursing (34.9 per cent), allied professionals (12.8 per cent), technicians (10.1 per cent), support staff (4.6 per cent), research (6.9 per cent), systems (1.4 per cent), and physicians (0.9 per cent). Their occupational status was further coded using a five category version of the Pineo system (Pineo, Porter, \& McRoberts, 1977). Categories in the Pineo system included: (1) selfemployed professionals, employed professionals, high-level management (12.0 per cent); (2) semiprofessionals, technicians, middle management (70.5 per cent); (3) supervisors, foreperson, skilled clerical, skilled crafts and trades, farmers (12.4 per cent); (4) semi-skilled clerical, semi-skilled manual (3.7 per cent); (5) unskilled clerical, unskilled manual, farm laborers (1.4 per cent).

Participants identified their ethnic background in words, by responding to the question 'With what ethnic background do you identify most?'. The self-selected labels were categorized into two categories. Category 1 referred to self-selected terms which included only the word(s) 'Canadian' or 'Caucasian' or 'White' or 'anglo-saxon' or 'French-Canadian' or 'francophone', referring to individuals who identified primarily with being Canadian, White, or speaking one of the two official languages of the country. Category 2 included terms containing any other words to describe their ethnicity or cultural background. Using the described system, 21 per cent of participants fell into the 'other' Category 2.

\section{Procedure}

Hospital employees were recruited using four strategies: poster advertisements throughout the hospital, flyers attached to their paycheques, flyers in human resource packages if they were pregnant, access to a booth of information close to their cafeteria (for a duration of 2-3 days). The study was advertised as a study focusing on work-family balance, with a special focus on pregnancy. Recruitment was targeted towards two groups: (1) any employees working at least an average of 21 hours/week at the health care facility; (2) pregnant employees working at least an average of 21 hours/week at the health care facility. Participation was voluntary, and interested employees were asked to contact the study coordinator by email or phone. The study coordinator then explained in detail that the study involved completing a questionnaire at home twice within 12 to 18 months for the nonpregnant employees, and three times within 12-18 months for the pregnant employees. Employees were encouraged to have their partners (if applicable) participate in the study.

All participants completed a consent form, including the participating partners. The study was approved by each hospital's Research Ethics Board.

\section{Design of the study}

Although participants were involved in a threewave longitudinal study, the current paper reports cross-sectional results of Wave 1 only. 


\section{Instrumentation}

Sociodemographic information. The following information was gathered: age, highest level of education achieved (categorical, with graduate education as reference category), personal income (categorical), family income (categorical), marital status (categorical), number of children, presence of children younger than 18 years old (dichotomous).

Work conditions. Work conditions were assessed with two instruments-the ERI scale (Siegrist \& Peter, 1996) and the JCQ (Karasek, 1985; Karasek et al., 1998).

The ERI examines three dimensions of workEffort, reward, and overcommitment with a 46-item scale. The ERI has traditionally been analyzed by examining the ratio of effort-reward - a ratio above one is considered to be indicative of a high level of work stress (Peter et al., 1998). Only the ERI ratio was used as a continuous variable in the analyses. The overcommitment subscale was not used, due to its high overlap with depressive symptoms and depressogenic cognitions. The effort subscale of the ERI has six items assessing time pressure, interruptions, responsibility, pressure to work overtime, physically demanding work, and increasing demands (alpha coefficient of 0.78 with this sample). The reward subscale has 11 items assessing monetary gratification, esteem reward, security, and career opportunities associated with work (alpha coefficient of 0.82 with this sample).

The JCQ measures demand and control characteristics of jobs. A 17-item version was used assessing the control dimension (nine itemsalpha coefficient of 0.83 with this sample) and the demand dimension (eight items-alpha coefficient of 0.78 with this sample). The dichotomous strain variable was used in this analyses: high demand/low control as opposed to all other quadrants, based on the median split of the distribution of demand and control. In addition, the JCQ social support scale of 11 items was used, which is a combination of the coworker support and the supervisor support subscales (see Table I for all alpha coefficients for remaining scales).

Work-family balance. The 14-item WorkFamily Spillover Scale (Grzywacz \& Marks, 2000) was used to measure NWFS, PWFS, NFWS, and PFWS. This measure reflects two important concepts which have gained accep-
Table I. Means, standard deviations (SD), range and reliability estimates for study variables.

\begin{tabular}{|c|c|c|c|}
\hline Variables & Means (SD) & Range & Alpha coefficients \\
\hline ERI ratio & $0.56(0.28)$ & $0.20-1.71$ & $\begin{array}{l}\text { Not available } \\
\text { (dichotomous } \\
\text { variable) }\end{array}$ \\
\hline JCQ strain & $0.24(0.43)$ & $0-1$ & $\begin{array}{l}\text { In text for each } \\
\text { subscale }\end{array}$ \\
\hline $\begin{array}{c}\text { JCQ social } \\
\text { support }\end{array}$ & $32.73(5.17)$ & $17-44$ & 0.84 \\
\hline NWFS & $11.53(2.35)$ & $4-18$ & 0.77 \\
\hline NFWS & $9.51(2.22)$ & $5-15$ & 0.69 \\
\hline PWFS & $8.71(2.07)$ & $3-15$ & 0.74 \\
\hline PFWS & $10.50(2.20)$ & $3-15$ & 0.74 \\
\hline CES-D & $12.50(10.29)$ & $0-53$ & 0.92 \\
\hline
\end{tabular}

tance in the field of work-family balance: Firstly, that work-to-family and family-to-work spillover are distinct constructs which must be evaluated separately, and secondly, that both negative and positive aspects of spillover should be evaluated separately as well. The Work-Family Spillover Scale was factorially-derived with a sample of 1986 Americans participating in a large survey.

Depressive symptomatology. Depressive symptoms, measured by this scale, should be distinguished from more minor experiences of transient unhappiness. The 20-item Center for Epidemiological Studies Depression (CES-D) scale (Radloff, 1977) was used to measure depressive symptomatology. It is designed to cover major aspects of depression, with an emphasis on affective and neuro-vegetative components. This instrument is not a diagnostic tool, but is rather a screening tool. Based on studies examining the instrument's sensitivity and specificity, a cut-off score of 16 is considered to strongly suggest the presence of depression (Radloff, 1977). The validity of this cut-off score has been examined using both a clinician-administered scale and a structured interview as the criterion indices of the presence of a clinical disorder of depression. When using the clinician-administered Raskin Depression Scale (Maier et al., 1988) in a community sample of 482 adults, a cut-off score of 16 had a sensitivity of 63.6 per cent for detecting major depression, and a specificity of 93.9 per cent (Myers \& Weissman, 1980). Using the structured interview Schedule for Affective Disorders and Schizophrenia (SADS-L) as a criterion, the cut-off score of 16 had a sensitivity of 64 per cent and a specificity of 94 per cent (Boyd et al., 1982). 


\section{Work conditions, work-family spillover, and depressive symptomatology}

The CES-D has adequate reliability with alpha coefficients ranging from 0.76 and 0.90 (Radloff, 1977). It is also the only non-interview based and self-report measure of depressive symptoms which has adequate sensitivity and specificity when using clinician-administered scale and structured interview criterion. The CES-D was used as a continuous measure in this study.

\section{Analytical strategy}

Preliminary analyses. To examine potential multicollinearity, the variance inflation factor (Neter, Wasserman, \& Kutner, 1985) was used, where a variable was considered to be highly multicollinear with other variables if the variance inflation factor was above 10 . The variance inflation factor was less than 10 for all variables indicating that multicollinearity amongst the study variables was not an issue.

Initial data exploration was conducted, by regressing each socio-economic/health variable on the outcome, and showed that from all socioeconomic variables considered, only education and having children under the age of 18 at home were significantly related to the outcome. These two socio-economic variables were therefore included in the subsequent main analyses.

Main analyses. Exploratory path analysis was used to examine the relationships between sociodemographic variables, work conditions, negative/positive work-to-family and family-towork spillover and depressive symptomatology. Path analysis allows the simultaneous estimation of several regression equations, and provides estimates of direct and indirect impact of one variable on another. A dependent variable in one equation can be an independent variable in another. As some of the dependent variables were non-normal, Maximum Likelihood parameter estimates were calculated with standard errors and a mean, and a variance adjusted chi-square statistic, that are robust to non-normality, using Mplus version 3.0 (Muthen \& Muthen, 2004). The hypothesized model was first run (Model IHypothesized). Paths that were not significant were deleted from that model, and nonhypothesized paths which were significant and were theoretically sound were added to the model (Kline, 2005; McCallum, 1995). The model was re-run (Model II-Intermediate). Mplus provides modification indices which show the improve- ment in the chi-square value that would be obtained if a path was added or if covariance was estimated freely in the model. The final model (Model III-Final) was run after correlating the residual terms of some of the dependent variables as suggested by the modification indices. As suggested by Bollen and Long (1993) several goodness-of-fit measures were used to test the fit of the models: chi-square, Comparative Fit Index (CFI), Tucker-Lewis Index (TLI), Standardized Root Mean Square Residual (SRMR), and Weighted Root Mean Square Residual (WRMR). For the final model all fit indices were within acceptable limits (Yu, 2002).

Sample size justification. It is recommended that the ratio of the number of participants to the number of parameters to be estimated be of 10 to 1 (Kline, 2005). However, a model with a ratio of 5 to 1 remains acceptable (Hatcher, 1994). This hypothesized model included 34 parameters and the final model included 32 parameters with a sample size of 218 , yielding a ratio of participants to parameter of 6 to 1 .

\section{Results}

\section{Preliminary analyses}

Means, standard deviations, ranges, and alpha coefficients of the measures used in the path model are shown in Table I. Inter-correlations among predictor variables are shown in Table II. Goodness-of-fit indices are found in Table III, with direct, indirect and total effects of variables found in Table IV. Correlations among predictor variables were low to moderate. The variance inflation factor was not higher than 2.5 for any of the study variables, indicating a low level of multicollinearity.

\section{Main analyses}

Sixty-eight participants (31.2 per cent) had a CES-D score of 16 or above, indicating a high likelihood of the presence of a depressive disorder.

Model L-Hypothesized. The full hypothesized model, with the three work condition variables-the effort-reward ratio, the JCQ strain, 
Table II. Correlations for the study predictor variables.

\begin{tabular}{|c|c|c|c|c|c|c|c|c|c|c|}
\hline Variables & 1 & 2 & 3 & 4 & 5 & 6 & 7 & 8 & 9 & 10 \\
\hline 1. Education & & & & & & & & & & \\
\hline 2. Child under 18 years & -0.13 & & & & & & & & & \\
\hline 3. ERI & $-0.18^{*}$ & -0.03 & & & & & & & & \\
\hline 4. JCQ strain & 0.11 & 0.12 & $0.42^{* * *}$ & & & & & & & \\
\hline 5. JCQ social support & 0.03 & -0.09 & $-0.47^{* * *}$ & $-0.42 * * *$ & & & & & & \\
\hline 6. NWFS & -0.01 & 0.09 & $0.49 * * *$ & 0.14 & $-0.35 * * *$ & & & & & \\
\hline 7. NFWS & -0.09 & $0.22 *$ & $0.30 * * *$ & 0.07 & $-0.19 * *$ & $0.50^{* * *}$ & & & & \\
\hline 8. PWFS & 0.16 & -0.03 & -0.03 & $-0.16^{*}$ & $0.14^{*}$ & -0.03 & 0.05 & & & \\
\hline 9. PFWS & 0.10 & $-0.34^{* * *}$ & $-0.14^{*}$ & -0.07 & $0.16^{*}$ & $-0.16^{*}$ & $-0.25 * * *$ & $0.25^{* * *}$ & & \\
\hline 10. CES-D & $-0.33 * *$ & $0.16^{*}$ & $0.36^{* * *}$ & $0.20^{*}$ & $-0.22 * *$ & $0.34^{* * *}$ & $0.24 * * *$ & -0.10 & $-0.27^{* * *}$ & \\
\hline
\end{tabular}

Note: Pearson, point-biserial, and tetrachoric correlations are reported as appropriate.

* $p<0.05 ; * *<0.01$.

Table III. Goodness-of-fit indices of models.

\begin{tabular}{lcclcccc}
\hline Models & $\chi^{2}$ & $\mathrm{df}$ & $p$-Value & TLI & CFI & SRMR & WRMR \\
\hline Model I-Hypothesized & 105.9 & 15 & 0.000 & 0.545 & 0.150 & 0.096 & 1.741 \\
Model II-Intermediate & 60.01 & 17 & 0.000 & 0.784 & 0.645 & 0.065 & 1.167 \\
Model III-Final & 20.18 & 16 & 0.2124 & 0.979 & 0.963 & 0.038 & 0.751 \\
\hline
\end{tabular}

$\chi^{2}=$ chi-square; $\mathrm{df}=$ degrees of freedom; TLI = Tucker-Lewis index; CFI = Comparative Fit Index; SRMR = Standardized Root Mean Square Residual; WRMR = Weighted Root Mean Square Residual.

Table IV. Standardized ${ }^{\dagger}$ direct, indirect and total effects of antecedent variables on depressive symptoms.

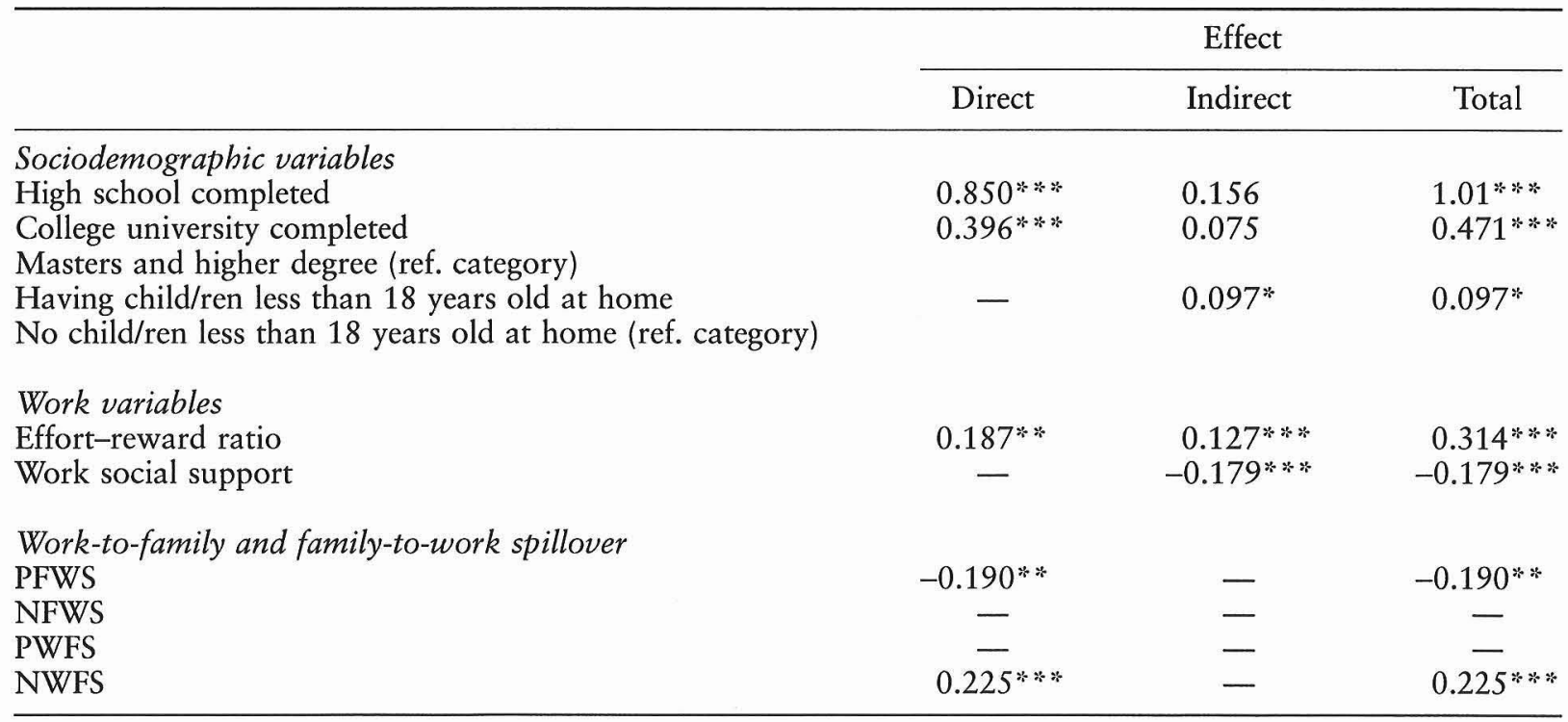

${ }^{t}$ For exogenous dummy variables standardized estimates were calculated using the standard deviation of the endogenous variables only. For continuous predictor variables both the standard deviations of the predictor and the outcome variables were used. - Indicates paths that were non-significant and therefore not estimated.

$* p<0.05 ; * * p<0.01 ; * * p<0.001$

and the JCQ social support-was initially run. Surprisingly, none of the three work condition measures was significantly associated with depressive symptomatology, indicating the possi- bility of high shared variance among the three measures (Table I). Given the cited conceptual overlap between the effort-reward ratio and the JCQ strain (Bosma, Peter, Siegrist, \& Marmot, 


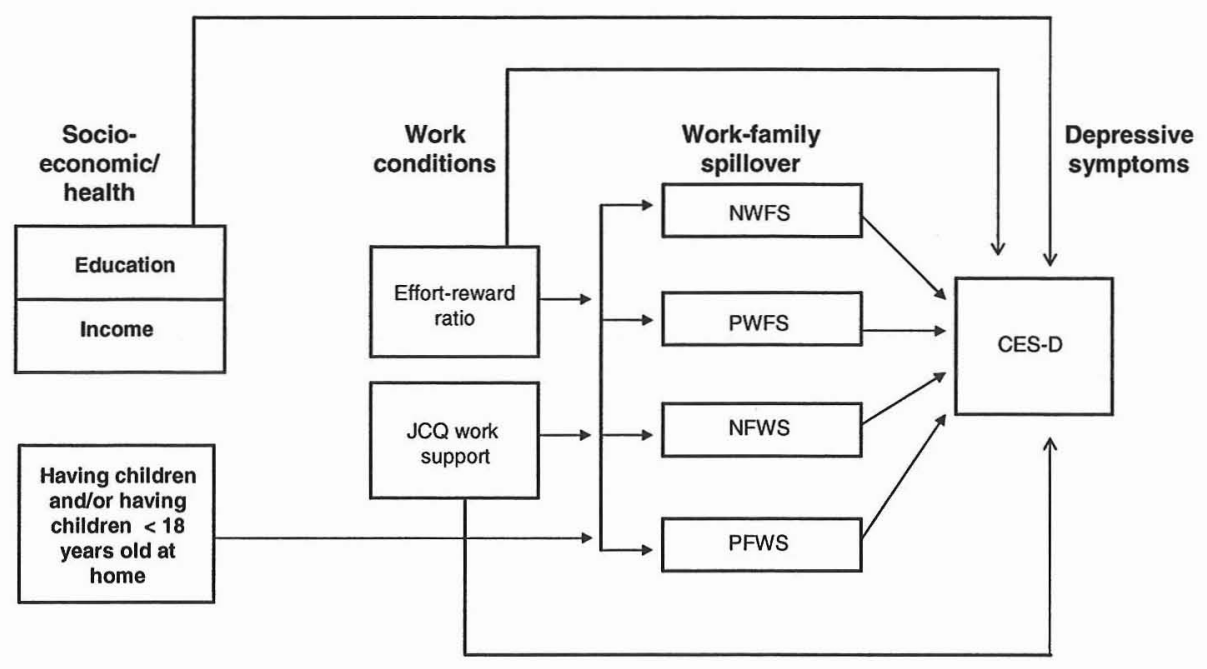

Figure 1. Model I-Hypothesized paths.

1998) and the statistically significant correlations between the two measures found in this study (see Table II), the model was subsequently run twiceby including each work condition measure separately. For the model with JCQ strain and JCQ social support included, there was no significant relationship between strain and depressive symptomatology. For the model with ERI ratio and JCQ social support, the relationship between the ERI ratio and depressive symptomatology was significant (estimate/standard error $=2.63, p<$ $0.01)$. Therefore we retained the model with the ERI ratio as the measure of work conditions. As can be seen in Table III, all four indices of goodness-of-fit for this model were poor, with the TLI under 0.95, the CFI under 0.95, the SRMR over 0.05 , and the WRMR over 0.9. Based on the findings of this first model, the following nonsignificant paths were eliminated from the subsequent model: children under 18-years-old to NWFS and PWFS; ERI ratio to PWFS; JCQ support to PFWS and NFWS; PWFS, NFWS and JCQ support to the CES-D. In addition, modification indices resulted in the addition of a path from JCQ support to ERI ratio.

Model II-Intermediate. The model was run without the non-significant paths identified in the previous model and with the added path from JCQ support to ERI ratio. All remaining paths in this model were significant. However, although the fit of this model was improved as compared to the hypothetical model, the fit remained poor (see Table III).
Model III-Final. This model kept the same paths as in Model II, but based on modification indices, correlated residuals between the errors of the following variables were added to the model: NWFS with NFWS, PWFS with PFWS. The inclusion of the correlated residuals between errors resulted in the path between the JCQ support and PWFS no longer being significant, and it was therefore dropped from the model. The final model resulted in a good fit: the TLI and the CFI were above 0.95, the SRMR was below 0.05, and the WRMR was below 0.9 (see Table III). Twenty-seven per cent of the variance in the CES$\mathrm{D}$ was explained. The standardized coefficients of statistically significant paths of Model III are found in Figure 2.

To explore hypothesized direct and mediational effects, the direct effects and the specific indirect effects were examined. For hypothesis (1), the direct effect from the ERI ratio to the CES-D was significant (estimate/standard error $=2.71, p<$ $0.015)$, but not the direct effect between the JCQ support and the CES-D. With the JCQ strain variable now eliminated from the model, the path between JCQ strain and CES-D was not supported either. For hypothesis (2), the mediational effects from the ERI ratio and the JCQ support to the CES-D were partially supported. Results showed that the effects of both the ERI ratio and the JCQ support on the CES-D were mediated by the NWFS (estimate/standard error $=3.63, p<$ 0.001 for the ERI ratio; estimate/standard error $=-2.00, p<0.05$ for JCQ support). Effects of JCQ support were significantly mediated by the 


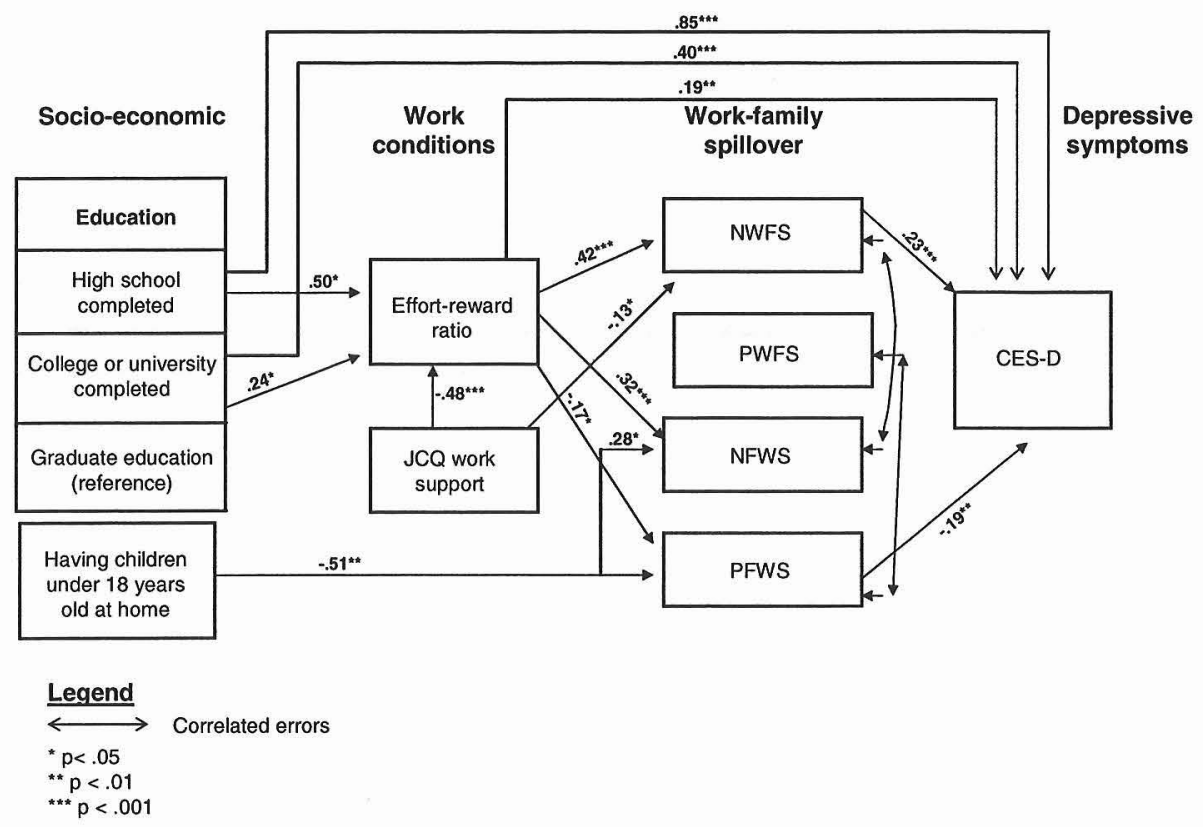

Figure 2. Model III-Final model.

ERI ratio (estimate/standard error $=-2.43, p<$ 0.05 ) and by the combined paths of the ERI ratio and the NWFS (estimate/standard error $=-3.50$, $p<0.001$ ). For hypothesis (3), a direct effect between education and CES-D was supported (estimate/standard error $=4.12, p<0.001$ for high school level education; estimate/standard error $=3.31, p<0.001$ for college or university level education). In addition, a direct effect between education and effort-reward ratio (estimate/standard error $=2.09, p<0.05$ for high school level education) was supported. For hypothesis (4), the mediational effects were partially supported: The effect of having children under 18 years old on the CES-D was significantly mediated by PFWS (estimate/standard error = $2.26, p<0.05$ ), but not by the three other spillover scales.

\section{Discussion}

In this study, a model of relationships between work conditions, work-family balance, and depressive symptomatology was developed and tested using path analysis. The final model supported the presence of a direct relationship between depressive symptoms and the following variables: high ERI, high NWFS, low PFWS, and low education. The impact of low support from work was indirect only, as mediated by NWFS, and by the combined ERI and NWFS. An indirect effect of high ERI on depressive symptoms was mediated by increased NWFS. The indirect effect of having children of 18 years or younger at home on depressive symptomatology was mediated by decreased PFWS. Finally, the indirect effect of low education on depressive symptoms was mediated by high ERI and high NWFS.

Indirect effects of work conditions on depressive symptoms were mediated by NWFS, while the indirect effect of the family situation (having younger children) on depressive symptoms was mediated by PFWS. The direction of the spillover effects is theoretically sound: work factors are involved in work-to-family effects, and family factors in family-to-work effects. The findings suggest that depressive symptoms may occur when negative aspects of work have a detrimental impact on family life. Conversely, individuals are least vulnerable to depressive symptoms when family life has beneficial effects on work. To the authors' knowledge, this study represents the first study supporting the presence of a relationship between work conditions and depressive symptoms which is mediated by work-family spillover.

The initial model included both the ERI and the JCQ (strain and social support) models. However, 


\section{Work conditions, work-family spillover, and depressive symptomatology}

the JCQ strain construct did not remain in the final model. This suggests the ERI may be more directly related to depressive symptomatology than JCQ strain. The ERI assesses efforts and rewards which are related to a sense of justice and equity, which may be more directly related to self-esteem and self-representation, than the work conditions assessed by the JCQ. Self-esteem and self-representation are associated with vulnerability to depression (Beck, Rush, Shaw, \& Emery, 1979), which could explain the closer relationship between the ERI with depressive symptoms, as compared to the JCQ.

The finding of a direct effect between the ERI ratio and depressive symptomatology is consistent with findings in previous studies (de Jonge et al., 2000; Fuhrer, Stansfeld, Chemali, \& Shipley, 1999; Wang \& Patten, 2001). However, its indirect effect mediated by work-family spillover represents a new finding in the literature. In addition, the absence of a direct effect between low social support at work and high depressive symptomatology is surprising given findings of previous studies supporting a strong relationship between the two constructs (Dormann \& Zapf, 1999; Fuhrer et al., 1999). In this sample, the impact of social support on depressive symptoms was instead mediated by ERI and NWFS. This finding suggests that social support received at work is associated with reductions in NWFS and a higher sense of reward from work.

In terms of sociodemographic factors, lower educational level and the presence of children under 18 years of age were associated with increased depressive symptomatology. The direct effect of education on depressive symptoms was strong and is consistent with previous literature where lower education is associated with a higher risk for presence, persistence, and new episodes of depression (Lorant et al., 2003). Of particular interest is the fact that an indirect effect of lower education on depressive symptoms was mediated by higher ERI. This indicates that part of the relationship between low education and depressive symptoms is related to the association of low education with jobs involving high ERI. The presence of children under the age of 18 years old had an indirect effect on increased depressive symptoms, which was mediated by reduced PFWS. This finding is consistent with previous literature showing that the benefits of working are offset by the demands of caring for young children, when examining general psychological distress as an outcome (Cleary, 1983; Emmons, Biernat, Tideje,
Lang, \& Wortman, 1990; Gottlieb, Kelloway, \& Martin-Matthews, 1996).

The prevalence of being at high risk for depression was remarkably high in this sample. It should be noted that the CES-D, used to measure depressive symptomatology, is a measure with high sensitivity, but lower specificity, hence resulting in high false-positive. This characteristic of the instrument could partially explain the high prevalence of risk for depression in this sample. While this finding is discrepant with the pointprevalence rate of 2 per cent for depression in the Canadian population (Patten, 2002), it is consistent with the high rates of psychological distress reported for health care workers and for nurses in particular, a trend which apparently runs across countries (Bellerose et al., 1995; Préville et al., 1991; Sauter et al., 1990). While psychological distress represents a broader category of symptoms than depressive symptoms, it is likely that depressed individuals score high on psychological distress measures, suggesting a strong relationship between the two constructs. The mechanisms for the high prevalence of psychological distress remain hypothetical-as compared to other types of work or as compared to 10 years ago, health care workers may encounter more emotional demands, may have higher caseloads, or less flexibility in schedule. Marmot, Siegrist, Theorell, and Feeney (1999) also noted that ERI is frequent in occupations involving many person-based interactions, such as in the health care sector.

Strengths of the study include a comprehensive conceptualization and measurement of constructs. Its novelty lies in the fact that both work conditions and work-family spillover were considered concurrently as modifiable workplace factors associated with depressive symptomatology, while controlling for confounding socioeconomic factors. In addition, the facilitative effects of work-to-family and family-to-work effects were considered, and both the ERI and the JCQ models were used. Well-validated measures of work conditions, work-family spillover, and depressive symptoms, were used. Finally, the sample was diverse in terms of occupational status and area of work within the health care sector.

The limitations of the study are associated with the fact that it was not possible to assess the representativeness of the sample, since the authors did not have access to data regarding the nonparticipants. Given the voluntary nature of par- 
ticipation, selection bias cannot be eliminated. However, regarding the primary outcome of depression, the high rates of depressive symptomatology are consistent with the observation that health care workers are particularly at risk for mental health disorders (Sauter et al., 1990) and emotional distress (Bellerose et al., 1995; Bourbonnais et al., 1999; Préville et al., 1991). The fact that the final sample only included women prevents the authors from making any inferences about impact of gender. As well, previous history of depression, which is a wellknown risk factor for future depression, was not assessed in this study. The statistical power was sufficient but limited, and an increased sample size would strengthen confidence in the modeling results. Finally, the cross-sectional nature of the study precludes any inference of causality. It also increases the impact of the potential respondent style bias of negative affectivity. Future studies should adopt a longitudinal design to examine the directionality of causation between depressive symptoms and family-to-work spillover.

The findings suggest that workplace factors associated with workers' mental health are not only related to the work conditions and environment but also to the interface of work with family. It highlights the importance for workplace leaders and decision-makers to consider all facets of workers' lives, both their occupational lives and the interface between their work and personal lives. Many of the workplace factors found to be associated with risk for depression are modifiable: Support from coworkers and supervisors to workers is a facet of workplace culture which can be modeled by leaders and encouraged at all levels of workplaces; support for work-family balance and attention to the needs of parents of young children can be incorporated in workplace culture and policies. These changes, such as child sick day leave and on-site daycare, are already being implemented in some workplaces.

It will be important to conduct future research with a wider range of occupations, and to examine interventions conducive to organizational changes addressing workplace factors associated with depression.

\section{References}

Baba, V.V., Jamal, M., \& Tourigny, L. (2003). Work and mental health: A decade in Canadian research. Canadian Psychology, 39, 94-107.
Beck, A.T., Rush, A.J., Shaw, B.F., \& Emery, G. (1979). Cognitive therapy of depression. New York: Guilford Press.

Bellerose, C., Lavallée, C., Chénard, L., \& Levasseur, M. (1995). How is health doing in 1992-1993? (report no. 1). Santé Québec: Ministry of Health and Social Services, Government of Quebec, Montreal, Qc.

Bollen, K.A., \& Long, J.S. (1993). Introduction. In K.A. Bollen, \& J.S. Long (Eds), Testing structural equation models (pp. 1-9). Newbury Park, CA: Sage Publications.

Bosma, H., Peter, R., Siegrist, J., \& Marmot, M. (1998). Two alternative job stress models and the risk of coronary heart disease. American Journal of Public Health, 88, 68-74.

Bourbonnais, R., Comeau, M., \& Vézina, M. (1999). Job strain and evolution of mental health among nurses. Journal of Occupational Health Psychology, 4, 95-107.

Broadhead, W.E., Blazer, D.G., George, L.K., \& Tse, C.K. (1990). Depression, disability days, and days lost from work in a prospective epidemiologic survey [see comments]. Journal of the American Medical Association, 264, 2524-2528.

Boyd, J.H., Weismann, M.M., Thompson, W.D., et al. (1982). Screening for depression in a community sample: Understanding the discrepancies between depression symptom and diagnostic scales. Archives of General Psychiatry, 39, 1195-1200.

Cleary, P.D. (1983). Sex difference in psychological distress among married people. Journal of Health and Social Behavior, 24, 111-121.

de Jonge, J., Bosma, H., Peter, R., \& Siegrist, J. (2000). Job strain, effort-reward imbalance, and employee well-being: A large-scale cross-sectional study [Review]. Social Science o Medicine, 50, 1317-1327.

Dormann, C., \& Zapf, D. (1999). Social support, social stressors at work, and depressive symptoms: Testing for main and moderating effects with structural equations in a threewave longitudinal study. Journal of Applied Psychology, 84, 874-884.

Emmons, C.A., Biernat, M., Tideje, L.B., Lang, E.L., \& Wortman, C.B. (1990). Stress, support, and coping among women professionals with preschool children. In J. Eckenrode, \& S. Gore (Eds), Stress between work and family (pp. 61-93). New York: Plenum.

Frone, M.R. (2003). Work-family balance. In J.C. Quick, \& L.E. Tetrick (Eds), Handbook of occupational health psychology (pp. 143-162). Washington, DC: American Psychological Association.

Frone, M.R., Russell, M., \& Barnes, G.M. (1996). Work-family conflict, gender, and health-related outcomes: A study of employed parents in two community samples. Journal of Occupational Health Psychology, 1, 57-69.

Frone, M.R., Russell, M., \& Cooper, M.L. (1997). Relation of work-family conflict to health outcomes: A four-year longitudinal study of employed parents. Journal of Occuptational and Organizational Psychology, 70, 325-335.

Fuhrer, R., Stansfeld, S.A., Chemali, J., \& Shipley, M.J. (1999). Gender, social relations and mental health: Prospective findings from an occupational cohort (Whitehall II study). Social Science \& Medicine, 48, 77-87.

Goldberg, R.J., \& Steury, S. (2001). Depression in the workplace: Costs and barriers to treatment. Psychiatric Services: A Journal of the American Psychiatric Association, 52, 1639-1643.

Gottlieb, B.H., Kelloway, E.K., \& Martin-Matthews, A. (1996). Predictors of work-family conflict, stress, and job satisfaction among nurses. Canadian Journal of Nursing Research, 28, 99-117. 


\section{Work conditions, work-family spillover, and depressive symptomatology}

Grzywacz, J.G., \& Bass, B.L. (2003). Work, family, and mental health: Testing different models of work-family fit. Journal of Marriage and the Family, 65, 248-262.

Grzywacz, J.G., \& Dooley, D. (2003). 'Good jobs' to 'bad jobs': Replicated evidence of an employment continuum from two large surveys. Social Science and Medicine, 56, 1749-1760.

Grzywacz, J.G., \& Marks, N.F. (2000). Reconceptualizing the work-family interface: An ecological perspective on the correlates of positive and negative spillover between work and family. Journal of Occupational Health Psychology, 5, 111-126.

Hatcher, L. (1994). A step-by-step approach to using the SAS system of factor analysis and structural equation modeling. Cary, NC: SAS Institute.

Johnson, J., Weissman, M.M., \& Klerman, G.L. (1992). Service utilization and social morbidity associated with depressive symptoms in the community. Journal of the American Medical Association, 267(11), 1478-1483.

Karasek, R.A. Jr. (1979). Job demands, job decision latitude, and mental strain: Implications for job redesign. Administrative Science Quarterly, 24, 285-308.

Karasek, R. (1985). Job Content Questionnaire and user's guide. [1.1]. Los Angeles, CA: University of Southern California.

Karasek, R., Brisson, C., Kawakami, N., Houtman, I., Bongers, P., \& Amick, B. (1998). The job content questionnaire (JCQ): An instrument for internationally comparative assessments of psychosocial job characteristics. Journal of Occupational Health Psychology, 3, 322-355.

Kelloway, E.K., Gottlieb, B.H., \& Barham, L. (1999). The source, nature, and direction of work and family conflict: A longitudinal investigation. Journal of Occupational Health Psychology, 4, 337-346.

Kessler, R.C., Andrews, G., Mroczek, D.K., Ustun, B., \& Wittchen, H.U. (1998). The World Health Organization Composite International Diagnostic Interview Short-Form (CIDI-SF). International Journal of Methods in Psychiatric Research, 7, 171-185.

Kline, R.B. (2005). Principles and practice of structural equation modeling (2nd ed.). New York: The Guilford Press.

Lorant, V., Deliege, D., Eaton, W., Robert, A., Philipot, P., \& Ansseau, M. (2003). Socioeconomic inequalities in depression: A meta-analysis. American Journal of Epidemiology, 157, 98-112.

Maier, W., Heuser, I., Philipp, M., et al. (1988). Improving depression severity assessment-II. Content, concurrent and external validity of three observer depression scales. Journal of Psychiaric Research, 22, 13-19.

Major, V.S., Klein, K.J., \& Ehrhart, M.G. (2002). Work time, work interference with family, and psychological distress. Journal of Applied Psychology, 87, 427-436.

Marmot, M., Siegrist, J., Theorell, T., \& Feeney, A. (1999). Health and the psychosocial environment and work. In M. Marmot, \& R.G. Wilkinson (Eds), Social determinants of bealth (pp. 105-131). Oxford: Oxford University Press.

Mausner-Dorsch, H., \& Eaton, W.W. (2000). Psychosocial work environment and depression: Epidemiologic assessment of the demand-control model. American Journal of Public Health, 90, 1765-1770.

McCallum, R.C. (1995). Model specification: Procedures, strategies, and related issues. In R.H. Hoyle (Ed.) (pp. 16-55). Thousand Oaks, CA: Sage Publications.

Muthen, L.K., \& Muthen, B.O. (2004). Mplus user's guide (3rd ed.). Los Angeles, CA: Muthen \& Muthen.

Myers, J.K., \& Weissman, M.M. (1980). Use of self-report symptom scale to detect depression in a community sample. American Journal of Psychiatry, 137, 1081-1084.
Neter, J., Wasserman, W., \& Kutner, M.H. (1985). Applied linear statistical models. Homewood, IL: Richard D. Irwin Inc.

Niedhammer, I., Goldberg, M., Leclerc, A., Bugel, I., \& David, S. (1998). Psychosocial factors at work and subsequent depressive symptoms in the Gazel cohort. Scandinavian Journal of Work, Environment and Health, 24, 197-205.

Paterniti, S., Niedhammer, I., Lang, T., \& Consoli, S.M. (2002). Psychosocial factors at work, personality traits and depressive symptoms. Longitudinal results from the GAZEL study. British Journal of Psychiatry, 181, 111-117.

Patten, S.B. (2002). Progress against major depression in Canada. Canadian Journal of Psychiatry, 47(8), 775-780.

Peter, R., Alfredsson, L., Hammar, N., Siegrist, J., Theorell, T., \& Westerholm, P. (1998). High effort, low reward, and cardiovascular risk factors in employed Swedish men and women: Baseline results from the WOLF study. Journal of Epidemiology of Community Health, 52, 540-547.

Pineo, P.C., Porter, J., \& McRoberts, H.A. (1977). The 1971 census and the socioeconomic classification of occupations. The Canadian Review of Sociology \& Anthropology, 14, 91-102.

Préville, M., Beauchemin, J., \& Potvin, L. (1991). Study of characteristics associated to stress among nurses. Maisonneuve Rosemont Hospital, Department of Community Health.

Radloff, L.S. (1977). The CES-D scale: A self-report depression scale for research in the general population. Applied Psychology Measurement, 1(3), 385-401.

Sauter, S.L., Murphy, L.R., \& Hurrell, J.J. (1990). Prevention of work-related psychological disorders. American Psychologist, 45, 1146-1158.

Siegrist, J., \& Peter, R. (1996). Measuring Effort-Reward Imbalance at work: Guidelines. Dusseldorf: University of Dusseldorf.

Simon, G.E., Barber, C., Birnbaum, H.G., Frank, R.G., Greenberg, P.E., Rose, R.M., et al. (2001). Depression and work productivity: The comparative costs of treatment versus nontreatment. Journal of Occupational \& Environmental Medicine, 43, 2-9.

Stansfeld, S.A., Fuhrer, R., Shipley, M.J., \& Marmot, M.G. (1999). Work characteristics predict psychiatric disorder: Prospective results from the Whitehall II Study. Occupational \& Environmental Medicine, 56, 302-307.

Stephens, T., \& Joubert, N. (2001). The economic burden of mental health problems in Canada. Chronic Disorders in Canada, 22, 18-23.

van der Doef, M., \& Maes, S. (1999). The job demand-control (-support) model and psychological well-being: A review of 20 years of empirical research. Work \& Stress, 13, 87-114.

Voydanoff, P. (2004). Implications of work and community demands and resources for work-to-family conflict and facilitation. Journal of Occupational Health Psychology, 9, 275-285.

Wall, T.D., Bolden, R.I., Borrill, C.S., Carter, A.J., Goyla, D.A., Hardy, G.E., et al. (1997). Minor psychiatric disorder in NHS trust staff: Occupational and gender differences. British Journal of Psychiatry, 171, 519-523.

Wang, J.L., \& Patten, S.B. (2001). Perceived work stress and major depression in the Canadian employed population, 20-49 years old. Journal of Occupational Health Psychology, 6, 283-289.

Yu, C.-Y. (2002). Evaluating cutoff criteria of model fit indices for latent variable models with binary and continuous outcomes. Doctoral dissertation, University of California, Los Angeles, CA. 